International Journal of Pure and Applied Mathematics

Volume 94 No. 3 2014, 373-377

ISSN: 1311-8080 (printed version); ISSN: 1314-3395 (on-line version)

url: http://www.ijpam.eu

doi: http://dx.doi.org/10.12732/ijpam.v94i3.6

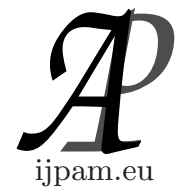

\title{
EQUIVALENCE OF TWO WIDELY RESEARCHED PROBLEMS IN HYPERGRAPH THEORY
}

\author{
R. Dharmarajan ${ }^{1}$, K. Kannan ${ }^{2}$ \\ ${ }^{1,2}$ Department of Mathematics \\ SASTRA University \\ Thanjavur, Tamilnadu State, INDIA
}

\begin{abstract}
The problems of enumerating (i) all the minimal transversals and (ii) all the minimal dominating sets, in a given hypergraph, have received a lot of attention because of their applications in Computer Science. This article explores the possibilities of these two problems being solution-wise equivalent - that is, each solution to one of them being a solution to the other - in the domain of Sperner hypergraphs, culminating in identifying the only class of such hypergraphs in which the equivalence holds.
\end{abstract}

AMS Subject Classification: 05C65

Key Words: hypergraph, hyperedge, Sperner hypergraphs, trim hypergraph, transversal, dominating set

\section{Introduction}

The cardinality (or, size) [8] of a finite set $V$ is denoted by $|V|$. The power set of $V$ is the set of all subsets (including the empty set $\phi$ ) of $V$, and is denoted by $2^{V}$. The set of all nonempty subsets of $V$ is denoted by $2^{V *}$; that is, $2^{V *}=$ $2^{V}-\{\phi\}$.

A simple hypergraph [2] is an ordered couple $H=(V, E)$ where: (i) $V$ is a nonempty finite set and (ii) $E$ is a set of nonempty subsets of $V$ such that

Received: January 2, 2014

(C) 2014 Academic Publications, Ltd.

$\S$ Correspondence author url: www.acadpubl.eu 
$\bigcup_{X \in E} X=V$. Each member of $V$ is a vertex in $H$; and each member of $E$ is a hyperedge (or, an edge) in $H$. A hyperedge $X$ with $|X|=1$ is a loop. $\mathrm{H}$ is loop-free if $|X|>1$ for every hyperedge $X$. Two distinct vertices $x$ and $y$ are adjacent if there is a hyperedge that contains both $x$ and $y . H$ is Sperner if no hyperedge is a subset of another. Sperner hypergraphs are necessarily simple, though not conversely [3].

A set $T \in 2^{V *}$ is a transversal [2] in $H$ if $T \cap X \neq \phi$ for each hyperedge $X$ (or, $T$ meets each of the hyperedges in $H$ ). A transversal $T$ is minimal if no proper subset of $T$ is a transversal.

A set $D \in 2^{V *}$ is a dominating set [1] in $H$ if either $D=V$ or each $y \in V-D$ is adjacent to some $x \in D$. A dominating set $D$ is minimal if no proper subset of $D$ is a dominating set.

The problem of identifying the minimal transversals in a given hypergraph $H$ will be denoted by $H Y P-T R A N S-H$, and the problem of identifying the minimal dominating sets in $H$ will be denoted by $H Y P-D O M-H$.

If $Y \in 2^{V *}$, then $Y$ is a solution to $H Y P-T R A N S-H$ (written $Y \in$ $H Y P-T R A N S-H)$ if $Y$ is a minimal transversal in $H$. And $Y$ is a solution to $H Y P-D O M-H$ (written $Y \in H Y P-D O M-H$ ) if $Y$ is a minimal dominating set in $H$.

The hypergraphs considered in this article are all assumed loop-free and of the Sperner type. A given hypergraph $H$ will be assumed to be the ordered couple $H=(V, E)$ unless alternate notations are explicit. This research is of theoretical interest, motivations for it coming from: (i) minimal transversals and related problems, dealt with in [4]; (ii) minimal dominating sets and related topics, covered in [6], and (iii) transversals and dominating sets, treated in [5].

The following polynomial-time equivalence is considered in [6]: If $P$ and $Q$ are enumeration problems for hypergraph properties $\alpha(X)$ and $\beta(X)$, respectively (where $X$ is a nonempty subset of vertices in the given hypergraph), then $P$ is at least as hard as $Q$ if an output-polynomial time algorithm for $Q$ implies an output-polynomial time algorithm for $P$; and $P$ is equivalent to $Q$ if each of the two enumeration problems is at least as hard as the other.

The equivalence considered in this article (Section 3), while being prompted by the one in [6], focuses on behaviour of solutions instead of their enumeration. So, the problems $H Y P-T R A N S-H$ and $H Y P-D O M-H$ have been viewed not as enumeration problems but, rather, as problems admitting common solutions under specific conditions. 


\section{Trim Hypergraphs}

Let $H=(V, E)$. A hyperedge $X$ is redundant in $H$ if $E-\{X\}$ covers $X$; that is, $X \subseteq \bigcup_{Y \in E-\{X\}} Y$. If $H$ has no redundant hyperedges then $H$ is a trim hypergraph. Evidently a trim hypergraph is Sperner, though not conversely. Trim hypergraphs are dealt with in some detail in [7].

Proposition 2.1. Every transversal is a dominating set, in any hypergraph.

Proposition 2.2. In a trim hypergraph $H$, a set $X \in 2^{V *}$ is a dominating set if and only if $X$ is a transversal.

Proposition 2.3. A Sperner hypergraph $H$ is trim if and only if every dominating set is a transversal.

Proofs of 2.1, 2.2 and 2.3 are given in [3].

Proposition 2.4. If $V$ is the minimal transversal in $H=(V, E)$, then $H$ is not loop-free.

Proof. If $|V|=1$ then the conclusion is obvious. In the case $|V|>1$, let $x, y \in V$ be distinct. Then $V-\{y\}$ is not a transversal, and so $\{y\}$ is a hyperedge in $H$.

\section{The Equivalence of HYP-TRANS-H and HYP-DOM-H in Trim Hypergraphs}

Let $H=(V, E)$ be a given hypergraph. Let $\alpha(X)$ and $\beta(X)$ be two different hypergraph properties. For instance, $\alpha(X)$ could be ' $X$ is a minimal dominating set'and $\beta(X)$ could be ' $X$ is a transversal.' Let $\alpha(H)$ denote the problem of identifying the sets $X$ (in $2^{V *}$ ) such that $\alpha(X)$ is true; and let $\beta(H)$ be the corresponding problem for $\beta(X)$. Then $\alpha(H)$ is solution-wise included in $\beta(H)$, written $\alpha(H) \prec \beta(H)$, if $\beta(X)$ is satisfied whenever $\alpha(X)$ is. $\alpha(H)$ and $\beta(H)$ are solution-wise equivalent, written $\alpha(H)(\equiv) \beta(H)$, if $\alpha(H) \prec \beta(H)$ as well as $\beta(H) \prec \alpha(H)$.

In 3.1 through $3.3, H$ is assumed Sperner and loop-free.

3.1: Proposition. If $H$ is trim, then $H Y P-T R A N S-H(\equiv) H Y P-$ $D O M-H$. 
Proof. Let $Y \in H Y P-T R A N S-H$. Then $Y$ is a proper subset of $V$ (by 2.4) and $Y$ is a dominating set (by 2.1). Were some proper subset $X$ of $Y$ a dominating set in $H$, then $X$ would be a transversal (by 2.3), contradicting $Y \in H Y P-T R A N S-H$. So $Y$ is a minimal dominating set in $H$, whence $H Y P-T R A N S-H \prec H Y P-D O M-H$.

On the other hand, let $Y \in H Y P-D O M-H$. Then $Y$ is a transversal in $H$ (by 2.3). Were some proper subset $X$ of $Y$ a transversal in $H$, then $X$ would be a dominating set (by 2.1), going against $Y \in H Y P-D O M-H$. So $Y$ is a minimal transversal in $H$, giving $H Y P-D O M-H \prec H Y P-T R A N S-H$ as well.

Proposition 3.2. If $H Y P-T R A N S-H(\equiv) H Y P-D O M-H$ then $H$ is trim.

Proof. Let $D$ be a given dominating set in $H$. Let $D_{1}$ be any minimal dominating set contained in $D$. Then, by hypothesis, $D_{1}$ is a minimal transversal in $H$ and so $D$ is a transversal in $H$. Then $\mathrm{H}$ is trim (by 2.3).

Propositions 3.1 and 3.2 imply the following proposition.

Proposition 3.3. $H Y P-T R A N S-H(\equiv) H Y P-D O M-H$ if and only if $H$ is trim.

\section{Resume}

The only class of loop-free Sperner hypergraphs in which the HYP-TRANS and HYP-DOM problems are solution-wise equivalent is the class of trim hypergraphs.

Consequently, enumerating the minimal transversals in a trim hypergraph $H$ is equivalent to enumerating the minimal dominating sets in $H$. A fortiori, an output-polynomial time algorithm for HYP-TRANS-H is one for HYP-DOM$\mathrm{H}$, and vice-versa. A future direction of research could be investigation of output-polynomial time algorithm to enumerate minimal transversals / minimal dominating sets in trim hypergraphs.

\section{Acknowledgements}

The authors express their thanks to: (1) Professor R. Sethuraman, Vice Chancellor, SASTRA University; (2) Dr. R. Balakrishnan, Adjunct Professor, Bharathi- 
dasan University, Tiruchirapalli (Tamilnadu, India); and (3) D. Ramachandran, Director, Abel-Jacobi Mathematics Foundation, Palghat (Kerala, India), for their suggestions and encouragement. The authors also gratefully acknowledge the funding provided by Abel-Jacobi Mathematics Foundation.

\section{References}

[1] B. D. Acharya, Domination in hypergraphs, AKCE Journal of Graphs and Combinatorics, 2 (2007), 117-126.

[2] C. Berge, Hypergraphs - Combinatorics on finite sets, North-Holland Mathematical Library, Amsterdam (1989).

[3] R. Dharmarajan and D.Ramachandran, On connections between dominating sets and transversals in simple hypergraphs, Int. J. Pure Appl. Math. 80 (5) (2012), 721-725.

[4] T. Eiter and G. Gottlob, Identifying the minimal transversals of a hypergraph and related problems, SIAM Journal on Computing, 24 (1995), 1278-1304.(doi: 10.1137/S0097539793250299)

[5] M. A. Henning, Transversals and domination in hypergraphs, 24th Cumberland Conference, University of Johannesburg (2011).

[6] Mamdou M. Kante, V. Limouzy, A. Mary and L. Nourine, Enumeration of minimal dominating sets and variants, LIMOS - Universite Blaise Pascal, GROW (2011).

[7] D. Ramachandran and R. Dharmarajan, On trimming simple hypergraphs, Global Journal of Science Frontier Research, 12 (9) (2012), 21-26.

[8] R. R. Stoll, Set Theory and Logic, Dover, New York (1963). 
